\title{
ORIGINAL ARTICLE \\ Do ambulatory patients with spinal cord injury walk symmetrically?
}

\author{
M Kumprou ${ }^{1,2}$, P Amatachaya ${ }^{2,3}$, T Sooknuan ${ }^{2,4}$, T Thaweewannakij ${ }^{1,2}$, L Mato $^{1,2}$ and S Amatachaya ${ }^{1,2}$
}

Study design: Cross-sectional study.

Objectives: To explore the levels of walking symmetry in ambulatory participants with spinal cord injury (SCI), and compare levels of walking symmetry among those with different degrees of lesion severity and those who walked with various types of walking devices. Setting: A tertiary rehabilitation center and many communities in Thailand.

Methods: Forty-six participants were assessed for their demographics, SCl characteristics and types of walking device used. Then they were recorded for the ability of walking over a 10-m walkway using a digital camera. The levels of walking symmetry were analyzed using a method of manual digitization and reported in terms of percents of step length ratio.

Results: Although the participants had bilateral sensorimotor impairments, they showed some degrees of walking asymmetry ( 90\%), particularly those with American Spinal Injury Association C (AIS C) (85\%) and those who walked with a standard walker (78\%). Their levels of walking symmetry also significantly differed from those with AIS D $(92 \% ; P<0.05)$ and those who walked without a walking device $(95 \% ; P<0.01)$.

Conclusions: Walking symmetry has been reported as an important variable for the ability of well-controlled walking. Therefore, apart from levels of independent walking, rehabilitation professionals need to emphasize improvements in walking symmetry among these individuals.

Spinal Cord (2017) 55, 204-207; doi:10.1038/sc.2016.149; published online 8 November 2016

\section{INTRODUCTION}

Sensorimotor impairments following spinal cord injury (SCI) reduce the walking ability of patients, and most ambulatory individuals can walk non-functionally (i.e., they are able to walk only within their house, or short distances, and require external assistance from persons or devices). ${ }^{1,2}$ Thus, improved quality and ability of ambulation is a common rehabilitative goal among these individuals. ${ }^{3,4}$

Among many variables relating to walking, walking symmetry has been emphasized as an important index for the ability of well-controlled walking in many groups of patients such as stroke and amputee..$^{5-7}$ With unilateral impairments, these patients commonly walk with a short step length of the non-affected side, to minimize the task demands on the affected limb during a single-support phase and quickly return to a more stable double-support phase. ${ }^{8}$ On the other hand, impairments in the affected limb also require a greater contribution from the non-affected limb (i.e., increased single-support time, weight-bearing ability and propulsive demands), which subsequently elevates the risk of musculoskeletal injury and articular joint degeneration. Additionally, the reduced use of the affected limb enhances the risk of muscular atrophy and bone density loss. ${ }^{9-11}$ Such asymmetrical walking manners require high metabolic demands, and they closely link to motor impairments that further affect important variables associated with the ability of community ambulation, such as walking speed and distance covered., ${ }^{9,12}$ Therefore, rehabilitation strategies by which to improve walking symmetry are important for these individuals. ${ }^{8}$

On the other hand, ambulatory patients with SCI likely have various degrees of bilateral, and sometime asymmetrical, sensorimotor impairments below the neurological level of the injury, depending on the site and severity of the lesion. ${ }^{3}$ With traditional concepts, some patients are trained to use a compensatory strategy and may require a walking device to compensate for the consequences of neurological deficits that may affect their levels of walking symmetry. ${ }^{13}$ However, to our knowledge, no evidence specifically reports levels of walking symmetry in ambulatory individuals with SCI. A study in this area would illustrate the pervasiveness of the problem and provide important clues as to how to improve rehabilitation strategies for these individuals. Thus, this study assesses levels of walking symmetry using percents of step length ratios in ambulatory patients with SCI, and compares the findings among those with various degrees of lesion severity and those who used various types of walking devices.

\section{MATERIALS AND METHODS \\ Participants}

Participants were cross-sectionally recruited from a tertiary rehabilitation center and from many communities in Thailand during the period of August 2015-April 2016. The inclusion criteria were having an incomplete SCI, with the American Spinal Cord Injury Association (ASIA) Impairment Scale or AIS

${ }^{1}$ School of Physical Therapy, Faculty of Associated Medical Sciences, Khon Kaen University, Khon Kaen, Thailand; ${ }^{2}$ Improvement of Physical Performance and Quality of Life (IPQ) Research Group, Khon Kaen University, Khon Kaen, Thailand; ${ }^{3}$ Department of Mechanical Engineering, Faculty of Engineering and Architecture, Rajamangala University of Technology Isan, Nakhon Ratchasima, Thailand and ${ }^{4}$ Department of Electrical Engineering, Faculty of Engineering and Architecture, Rajamangala University of Technology Isan, Nakhon Ratchasima, Thailand

Correspondence: Dr S Amatachaya, School of Physical Therapy, Faculty of Associated Medical Sciences, Khon Kaen University, Mittraphap Highway, Maung, Khon Kaen 40002, Thailand.

E-mail: samata@kku.ac.th

Received 30 May 2016; revised 17 September 2016; accepted 24 September 2016; published online 8 November 2016 
$\mathrm{C}$ or $\mathrm{D}$, and the ability of independent walking with or without a walking device for at least $15 \mathrm{~m}$ (Functional Independent Measure Locomotion (FIM-L) score $=5-7$ ). The patients were excluded if they had any other condition that might affect walking, such as muscular or joint pain with a pain score of at least 5 out of 10 on a visual analog scale, an obvious leg length discrepancy or inflammation in the joints of the lower limbs. ${ }^{14-16}$ The sample size was estimated based on that required for a major study on the importance of walking symmetry in ambulatory patients with SCI, with the $\alpha$ set to 0.05 and the power of the test set to $0.1 .^{17}$ The participants needed to read and sign written informed consent documents approved by the ethics committee of Khon Kaen University (HE581392) before taking part in the study.

\section{Research protocol}

The eligible participants were interviewed and assessed for their demographics (i.e., age, gender, weight and height), SCI characteristics, including causes (traumatic and nontraumatic), stages of SCI (subacute (postinjury time: PIT $<12$ month) and chronic (PIT $\geqslant 12$ months), severity and level of injury as determined using the sensorimotor scores and criteria from the standard ASIA protocol $^{18}$ and baseline walking ability (ability to walk with or without a walking device for at least $15 \mathrm{~m}$ ).

\section{Outcome of the study}

A recent review article on poststroke walking asymmetry suggested the use of spatiotemporal data (such as step length, swing time and stance time ratios) to quantify levels of walking symmetry. ${ }^{19}$ Many studies also applied these spatiotemporal parameters to report levels of walking symmetry because the findings were related to motor control and compensatory strategies during walking. ${ }^{8,10,11,19,20}$ Balasubramanian et al. ${ }^{20}$ further reported the importance of step length symmetry as it associated with variables relating to the ability of well-controlled walking. Consequently, the present study quantified levels of walking symmetry using percents of step length ratios.

A digital camera (Nikon D5300 with a $60-\mathrm{Hz}$ frame rate) was used to record a step length of participants while walking over a $10-\mathrm{m}$ walkway. before the test, the system was calibrated using an object of known length, and the three consecutive step lengths of each lower extremity were recorded over the $4 \mathrm{~m}$ in the middle of the walkway. Then the data were analyzed using a method of manual digitization by counting the pixels between the distance from the heel of one leg to the heel of the other leg and scaling with respect to the calibration object. This method showed high correlation with the PEAK motion analysis $(r=0.99$, ICC $3,1=0.99),{ }^{21}$ and it is practical to be applied in a rehabilitation ward and community. A value of $100 \%$ represents perfect walking symmetry, in which a participant might walk with either relatively longer less-affected or more-affected steps. ${ }^{20}$ Thus, the average step length of each lower extremity was

\section{Table 1 Demographics and $\mathrm{SCl}$ characteristics}

\begin{tabular}{lc}
\hline Variable & SCl participants $(\mathrm{n}=46)$ \\
\hline Age (years), mean \pm s.d. $(95 \% \mathrm{Cl})$ & $50.0 \pm 14.6(45.6-54.3)$ \\
Postinjury time (months), mean \pm s.d. $(95 \% \mathrm{Cl})$ & $40.5 \pm 44.3(27.4-53.7)$ \\
Gender: male ${ }^{\mathrm{a}}, n(\%)$ & $35(76.1)$ \\
Cause: traumatic SCla,$n(\%)$ & $23(50.0)$ \\
Level of injury: incomplete paraplegiaa,$n(\%)$ & $34(73.9)$ \\
Severity of injury: AIS Da, $n(\%)$ & $32(69.6)$ \\
Stage of injury: chronic ${ }^{\mathrm{a}}, n(\%)$ & $39(84.8)$ \\
Using a walking device, $\mathrm{n}(\%)$ & \\
$\quad$ Walker & $13(28.3)$ \\
Crutches & $5(10.9)$ \\
Cane & $7(15.2)$ \\
None & $21(45.7)$
\end{tabular}

Abbreviations: AIS, American Spinal Injury Association (ASIA) impairment scale; Cl, confidence interval; $\mathrm{n}$, number; $\mathrm{SCl}$, spinal cord injury.

Gender: male/female; cause of injury: traumatic SCl/nontraumatic SCl; level of injury: incomplete paraplegia/incomplete tetraplegia; severity of injury: AIS C or D; stage of injury: chronic/subacute.

aNote: These variables were categorized according to the following criteria. subsequently calculated using the following formula to minimize artificial inflation of denominator: ${ }^{10,14-16,19,21,22}$

$$
\text { Walking symmetry }(\%)=\frac{\text { Average data of the shorter step length }}{\text { Average data of the longer step length }} \times 100
$$

Participants were assessed when they walked without shoes, to minimize the effects of their shoes on the outcomes. To ensure their safety, they needed to fasten a lightweight safety belt around their waist and have an assessor walking or standing alongside them.

\section{Statistical analysis}

Descriptive statistics were used to explain the demographics, SCI characteristics and findings of the study. As the data were not normally distributed, the Mann-Whitney $U$-test and Kruskal-Wallis tests were used to compare the findings of those with different degrees of lesion severity (AIS C and D), and those who walked with various types of walking devices. A $P$-value $<0.05$ was considered statistically significant.

\section{RESULTS}

\section{Participant characteristics}

Forty-six ambulatory participants with SCI were involved in this study. Most of them were men at a chronic stage of SCI, and with rather good walking ability (i.e., able to walk without a walking device for at least $50 \mathrm{~m}$, or FIM-L score $=7$; Table 1). Most participants had bilateral asymmetrical sensorimotor deterioration, in which the significant difference between the more- and less-affected sides was found only in the lower-limb motor scores $(P=0.005$; Table 2$)$.

\section{Percents of walking symmetry among the participants}

The median walking symmetry among all the participants was $~ 90 \%$ (interquartile range (IQR): 79-96\%) when they walked at a preferred speed. When they walked at the fastest speed, the participants could increase the length of their shorter step by $12.05 \%$ and that of the longer step by $7.8 \%$, and they could increase levels of walking symmetry to 93\% (IQR: $83-97 \%$ ). At the fastest speed, participants with AIS C and D, and those who walked with various types of walking devices also increased the levels of walking symmetry, but this increment was not a significant difference $(P>0.05)$. Exceptionally for those who did not use a walking device, these participants showed significantly increased levels of asymmetry when they walked at the fastest speed ( $P=0.049$; Figure 2$)$.

In subgroup analysis, the data illustrated that participants with AIS C (median $=85 \%$ (IQR: $59-91 \%$ ) at the preferred speed, and $85 \%$ (IQR: $52-96 \%$ ) at the fastest speed) walked with a significantly greater

\section{Table 2 Sensorimotor scores of participants (according to ASIA} protocol)

\begin{tabular}{lccc}
\hline Variable $^{\mathrm{a}}$ & More-affected side & Less-affected side & P-value \\
\hline Motor UE (25 scores) & $23.67 \pm 2.65$ & $24.26 \pm 1.76$ & 0.214 \\
Motor LE (25 scores) & $15.96 \pm 5.62$ & $19.35 \pm 5.58$ & $0.005^{\mathrm{c}}$ \\
Light-touch UE (38 scores) & $35.26 \pm 6.10$ & $35.41 \pm 6.09$ & 0.905 \\
Light-touch LE (18 scores) & $11.83 \pm 7.10$ & $12.33 \pm 7.24$ & 0.739 \\
Pinprick UE (38 scores) & $35.37 \pm 6.09$ & $35.41 \pm 6.09$ & 0.973 \\
Pinprick LE (18 scores) & $11.83 \pm 7.10$ & $12.26 \pm 7.22$ & 0.771
\end{tabular}

Abbreviations: ASIA, American Spinal Cord Injury Association; LE, lower extremity; UE, upper

extremity.
Note: The data are mean \pm s.d.

Note: The data are mean \pm s.d.
a Assessed using criteria from the ASIA international standards for neurological classification of the five key muscles of the UE, five key muscles of the LE and 28 dermatomes of UE, LE and trunk. ${ }^{18}$

${ }^{b} P$-value derived from independent-sample $t$-test.

Indicates a significant difference. 


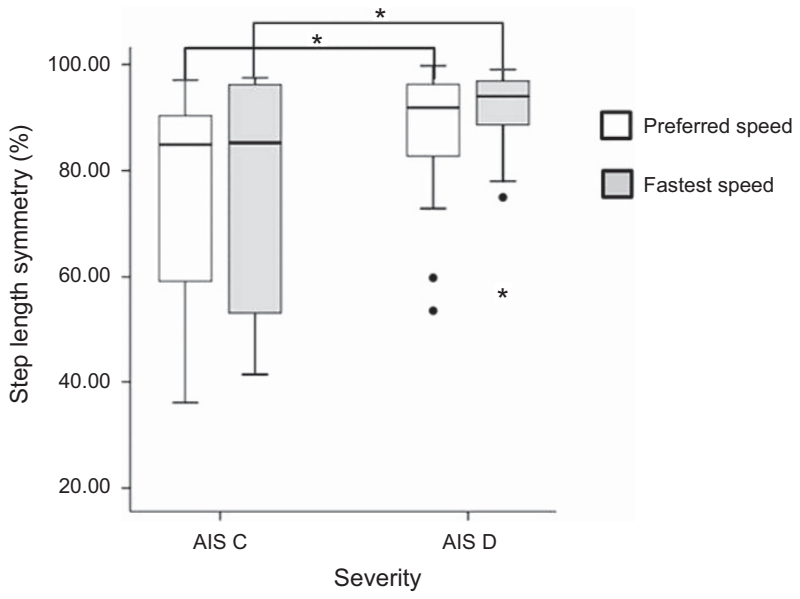

Figure 1 Percents of step length symmetry of participants with different levels of severity of $\mathrm{SCl}$, assessed using the preferred and fastest walking speed. Note: Comparisons were analyzed using the Mann-Whitney U-test. $* P<0.05$.

level of asymmetry compared with those with AIS D (median $=92 \%$ (IQR: $83-97 \%$ ) at the preferred speed, and 94\% (IQR: 88-97\%) at the fastest speed) $(P<0.05$; Figure 1$)$. In addition, asymmetrical walking was obvious among those who walked with a standard walker (median $=78 \%$ (IQR: $59-90 \%$ ) at a preferred walking speed, and 85\% (IQR: $51-93 \%$ ) at the fastest speed), followed by those who used a single cane $($ median $=90 \%$ (IQR: $79-92 \%)$ at a preferred speed, and 97\% (IQR: 92-98\%) at the fastest speed), crutches (median $=92 \%$ (IQR: $85-95 \%$ ) at a preferred speed, and 96\% (IQR: 82-97\%) at the fastest speed) and those who did not use a walking device $($ median $=95 \% \quad($ IQR: $85-98 \%)$ at a preferred speed, and $94 \%$ (IQR: $84-96 \%$ ) at the fastest speed) (Figure 2). At a preferred walking speed, the significant differences were found between those with AIS C and $\mathrm{D}(P<0.05$; Figure 1$)$ and those who walked with a standard walker and did not use a walking device $(P=0.009$; Figure 2$)$. At the fastest speed, levels of walking symmetry of those who used a standard walker were also significantly different from those who used a single cane $(P<0.05$; Figure 2$)$.

\section{DISCUSSION}

Healthy individuals are considered to walk symmetrically, with a normative range for spatiotemporal symmetry is assumed to be $0.9-1.1(90-110 \%)$, or the interlimb differences are $<6 \%{ }^{10,23}$ However, the existing evidence on asymmetrical walking is commonly involved in individuals with unilateral impairments such as stroke and amputee. ${ }^{7,8,20,23-25}$ The current findings confirm asymmetrical walking found in ambulatory participants with SCI who had bilateral sensorimotor impairments (78-94\%; Figure 2). The levels of their asymmetrical walking were at approximately the same levels as those seen in patients with stroke $(79-93 \%)^{9,20,23}$ and amputees (93.5\%). ${ }^{7}$ A possible explanation for the findings may be that although the participants had bilateral involvements, most of them had some degrees of asymmetrical lower-limb motor functionality and required a walking device $(P<0.01$; Table 2). The different impairments between the lower limbs and use of a walking device are important causes of asymmetrical walking in patients with neurological disorders. ${ }^{23}$ If participants were to persist in using such a walking manner, they would learn to use the limbs asymmetrically, which could subsequently increase their risk of musculoskeletal pain and joint damage in the limb of greater use, along with an increased risk of

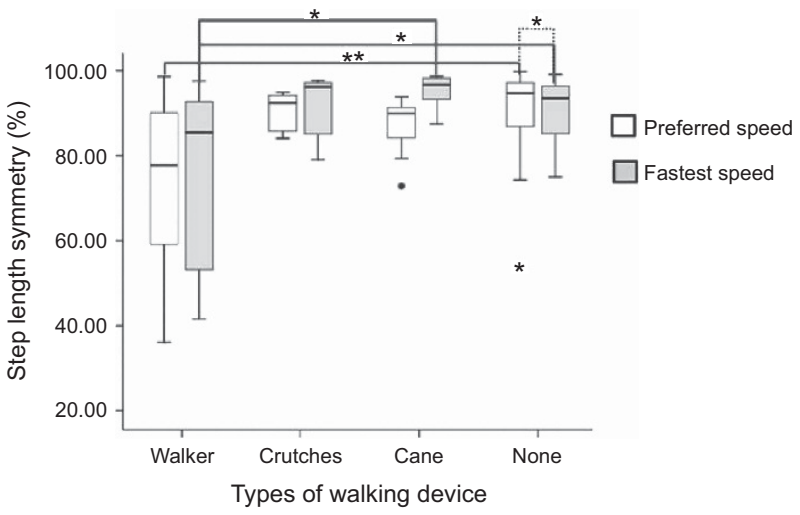

Figure 2 Percent of step length symmetry of the participants with different types of walking device, assessed using the preferred and fastest walking speed. Note: Comparisons were analyzed using the Kruskal-Willis test. ${ }^{*} P<0.05,{ }^{* *} P<0.01$.

muscular weakness and atrophy, as well as bone density loss, in the limb of lesser use. ${ }^{9,10}$

Further analysis indicated that the degrees of asymmetrical walking were obviously demonstrated in those with AIS C and those who walked with a standard walker (Figures 1 and 2). Having AIS C infers that the participants had severe sensorimotor deterioration (i.e., more than one-half of the key muscles below the neurological level have a muscle grade of $<3$ ). Thus they had poor prognosis for walking ability and needed alternative or compensatory strategies to execute a task, which further adversely affected the development of normal walking. ${ }^{1,3,26-28}$ In addition, a high proportion (54\%) of these participants needed a walking device to compensate for some of the impairments underlying the disability. The use of a walking device can adversely affect spatiotemporal variables relating to walking (i.e., step length, velocity and symmetry), particularly a more support device. ${ }^{28}$ With a wide base of support, a standard walker enhances stability, a sense of security and the ability to bear more weight on the upper extremities. ${ }^{29,30}$ However, the four-legged walker considerably limited a step dimension, and a patient likely adopted a step-to pattern that promoted asymmetrical step length. ${ }^{29}$ These could possibly explain the obvious asymmetrical manner among participants with an AIS C and those who used a standard walker (median $=78 \%$ (IQR: $59-90 \%$ ); Figure 2). For a single cane, it is often prescribed in a patient with rather good walking ability with the major aim of promoting self-confidence while walking. However, it allows for asymmetrical use of the upper extremities, which may affect levels of walking symmetry to a greater extent than that seen among those who used crutches (Figure 2).

When walking at the fastest speed, however, participants (except those who walked without any devices) could modify their step dimension and improve the level of walking symmetry. The findings may suggest the influence of the use of a walking device on the levels of walking symmetry and imply that these participants had residual capacity. With increased walking speed, the participants were facilitated to use their optimal ability, in particular, the more-affected limb that helped them improve the level of walking symmetry (Figure 2). Nonetheless, increased walking speed significantly reduced levels of walking symmetry among those who did not use any walking device. These findings align with those found in healthy individuals, 
and they suggest the effects of inherent neuromuscular asymmetry among the participants. ${ }^{31}$

Nonetheless, some limitations need to be considered when interpreting the findings. First, this study was cross-sectionally conducted, and there were only a few participants who walked with crutches (11\%) and a cane (15\%). Each group with different lesion severity and type of walking device used also contained participants with heterogeneous characteristics. Although the proportion of walking device used was coherent with the nature of ambulatory patients with SCI reported in a previous study, ${ }^{28}$ such characteristics may affect data comparisons among the groups and limit the generalizability of the findings. Furthermore, the findings illustrate the magnitude, but not impact, of asymmetrical walking for these individuals. Therefore, a further study should be undertaken in a greater number of participants, and examine the impacts of asymmetrical walking or effects of intervention to promote symmetrical walking to strengthen the importance of this variable for these patients.

\section{CONCLUSION}

The current findings confirm problems of asymmetrical walking among ambulatory individuals with SCI who have bilateral sensorimotor impairments, particularly those with AIS C (85\%) and those who use a standard walker $(78 \%)$. As walking symmetry is important for the ability of efficient and well-controlled walking, apart from the ability of independent walking, rehabilitation professionals may need to emphasize improving walking symmetry in these individuals.

\section{DATA ARCHIVING}

There were no data to deposit.

\section{CONFLICT OF INTEREST}

The authors declare no conflict of interest.

\section{ACKNOWLEDGEMENTS}

We thank for funding support from the Research and Researcher for Industry (RRi) (MSD58I0118) Postgraduate School of Khon Kaen University and the Improvement of Physical Performance and Quality of life (IPQ) research group, Khon Kaen University, Thailand.

1 Brotherton SS, Krause JS, Nietert PJ. Falls in individuals with incomplete spinal cord injury. Spinal Cord 2007; 45: 37-40.

2 Amatachaya S, Pramodhyakul W, Srisim K. Failures on obstacle crossing task in independent ambulatory patients with spinal cord injury and associated factors. Arch Phys Med Rehabil 2015; 96: 43-48.

3 Kirshblum SC, Priebe MM, Ho CH, Scelza WM, Chiodo AE, Wuermser LA. Spinal cord injury medicine. 3. rehabilitation phase after acute spinal cord injury. Arch Phys Med Rehabil 2007; 88: 62-70.

4 Sharp KG, Gramer R, Butler L, Cramer SC, Hade E, Page SJ. Effect of overground training augmented by mental practice on gait velocity in chronic, incomplete spinal cord injury. Arch Phys Med Rehabil 2014; 95: 615-621.

5 Gouwanda D, Senanayake A. Identifying gait asymmetry using gyroscopes: a crosscorrelation and Normalized Symmetry Index approach. J Biomech 2011; 44: 972-978.
6 Kaufman KR, Frittoli S, Frigo CA. Gait asymmetry of transfemoral amputees using mechanical and microprocessor-controlled prosthetic knees. Clin Biomech 2012; 27 460-465.

7 Savin DN, Morton SM, Whitall J. Generalization of improved step length symmetry from treadmill to overground walking in persons with stroke and hemiparesis. Clin Neurophysiol 2014; 125: 1012-1020.

8 Alexander LD, Black SE, Patterson KK, Gao F, Danells CJ, Mcllroy WE. Association between gait asymmetry and brain lesion location in stroke patients. Stroke 2009; 40: 537-544.

9 Patterson KK. Gait Asymmetry Post-Stroke. Department of Rehabilitation Science: University of Toronto, ON, Canada, 2010.

10 Patterson KK, Parafianowicz I, Danells CJ, Closson V, Verrier MC, Staines WR et al. Gait asymmetry in community-ambulating stroke survivors. Arch Phys Med Rehabil 2008; 89: 304-310.

11 Patterson KK. Evaluation of gait symmetry after stroke: a comparison of current methods and recommendations for standardization. Gait Posture 2010; 31: 241-246.

12 Zorner B, Dietz V, Curt A. Clinical algorithm for predicting walking capacity based on the ASIA motor score in acute SCI. Paper presented at Combined ASIA/ISCOS Meeting, Boston, MA, USA, 2006.

13 Behrman AL, Bowden MG, Nair PM. Neuroplasticity after spinal cord injury and training: An emerging paradigm shift in rehabilitation and walking recovery. Phys Ther 2006; 86: 1406-1425.

14 Pramodhyakul N, Amatachaya P, Sooknuan T, Arayawichanon P, Amatachaya S. Effects of a visuotemporal cue on walking ability of independent ambulatory subjects with spinal cord injury as compared with healthy subjects. Spinal Cord 2014; 52: 220-224.

15 Amatachaya S, Amatachaya P, Keawsutthi M, Siritaratiwat W. External cues benefit walking ability of ambulatory patients with spinal cord injury. Spinal cord 2013; 36 638-344.

16 Amatachaya S, Keawsutthi M, Amatachaya P, Manimmanakorn N. Effects of external cues on gait performance in independent ambulatory incomplete spinal cord injury patients. Spinal Cord 2009; 47: 668-673.

17 Portney L, Watkins M. Foundation of Clinical Research: Applications to Practice, , 3rd edn. Pearson Prentice-Hall: Englewood Cliffs, NJ, USA, 2009.

18 Kirshblum SC, Burns SP, Biering-Sorensen F, Donovan W, Graves DE, Jha A et al. International standards for neurological classification of spinal cord injury [revised 2011]. Spinal Cord Med 2011; 34: 535-546.

19 Lauzière S, Betschart M, Aissaoui R, Nadeau S. Understanding spatial and temporal gait asymmetries in individuals post stroke. Int J Phys Med Rehabil 2014; 2: $1-11$.

20 Balasubramanian CK, Bowden MG, Neptune RR, Kautz SA. Relationship between step length asymmetry and walking performance in subjects with chronic hemiparesis. Arch Phys Med Rehabil 2007; 88: 43-49.

21 Said CM, Goldie PA, Patla AE, Sparrow WA. Effect of stroke on step characteristics of obstacle crossing. Arch Phys Med Rehabil 2001; 82: 1712-1719.

22 Poncumhak P, Saengsuwan J, Kamruecha W, Amatachaya S. Reliability and validity of three functional tests in ambulatory patients with spinal cord injury. Spinal Cord 2013; 51: 214-217.

23 Hsu A-L, Tang P-F, Jan M-H. Analysis of impairments influencing gait velocity and asymmetry of hemiplegic patients after mild to moderate stroke. Arch Phys Med Rehabil 2003; 84: 1185-1193.

24 Schaarschmidt M, Lipfert SW, Meier-Gratz C, Scholle HC, Seyfarth A. Functional gait asymmetry of unilateral transfemoral amputees. Hum Movement Sci 2012; 31. 907-917.

25 Tura A, Raggi M, Rocchi L, Cutti AG, Chiari L. Gait symmetry and regularity in transfemoral amputees assessed by trunk accelerations. J Neuroeng Rehabil 2010; 7 $1-10$

26 Scivoletto G, Di Donna V. Prediction of walking recovery after spinal cord injury. Brain Res Bull 2009; 78: 43-51.

27 Scivoletto G, Romanelli A, Mariotti A, Marinucci D, Tamburella F, Mammone A et al. Clinical factors that affect walking level and performance in chronic spinal cord lesion patients. Spine 2008; 33: 259-264.

28 Saensook W, Phonthee S, Srisim K, Wattanapan P, Amatachaya S. Ambulatory assistive devices and walking performance in patients with incomplete spinal cord injury. Spinal Cord 2013, 1-4.

29 Bateni H, Maki BE. Assistive devices for balance and mobility: benefits, demands, and adverse consequences. Arch Phys Med Rehabil 2005; 86: 134-145.

30 Melis EH, Torres-Moreno R, Barbeau H, Lemaire ED. Analysis of assisted-gait characteristics in persons with incomplete spinal cord injury. Spinal Cord 1999; 37: 430-439.

31 Seeley K, Umberger BR, Shapiro R. A test of the functional asymmetry hypothesis in walking. Gait Posture 2008; 28: 24-28. 